PROCEEDINGS OF THE

AMERICAN MATHEMATICAL SOCIETY

Volume 36, No. 2, December 1972

\title{
A THEORY OF GRADE FOR COMMUTATIVE RINGS ${ }^{1}$
}

\author{
S. FLOYD BARGER
}

ABStract. A theory of grade is developed using $R$-sequences, the Koszul complex and standard homological algebra. Most results of interest are for finitely generated ideals.

The theory of grade as developed in [5] or [7] is restricted to Noetherian rings. In all that follows rings are commutative with identity and modules are unitary; however no chain conditions are assumed. This theory has been applied in [1] to generalize [6].

Three notions of the grade of an ideal appear in the non-Noetherian case. These are denoted $\mathrm{c}$ gr, $\mathrm{k}$ gr, and $\mathrm{r}$ gr as defined later. A slightly stronger version of the following will be established.

THEOREM. If $I$ is a finitely generated ideal and $E$ is an $R$-module with $I E \neq E$, then $\mathrm{c} \operatorname{gr}(I, E) \leqq \mathrm{k} \operatorname{gr}(I, E) \leqq \mathrm{r} \operatorname{gr}(I, E)$.

1. Koszul complex and grade. Let $I$ be an ideal in $R$ and $E$ an $R$-module with $I E \neq E$. Let $\mathrm{c} \operatorname{gr}(I, E)$ be the length of the longest maximal $R$-sequence on $E$ in $I$. For $\left\{x_{1}, \cdots, x_{s}\right\}$ a finite subset of $I$, let $g\left(x_{1}, \cdots, x_{s} \mid E\right)=s-t$ where $t$ is the largest integer so that the $t$ th homology module of the Koszul complex over $E$ determined by $x_{1}, \cdots, x_{s}$ is not zero. Let $\mathrm{k} \operatorname{gr}(I, E)$ be the supremum over all finite subsets $\left\{x_{1}, \cdots, x_{n}\right\}$ of the integers $g\left(x_{1}, \cdots, x_{n} \mid E\right)$. For $R$ Noetherian, $\mathrm{c} \operatorname{gr}(I, E)=\mathrm{k} \operatorname{gr}(I, E)$. The common value is the grade of $I$ on $E$. The first proposition follows from the properties of the Koszul complex [7].

Proposition 1. Let $I$ and $J$ be ideals, $E$ an $R$-module with $J E \neq E$. Then

(1) If $I \subseteq J, \mathrm{k} \operatorname{gr}(I, E) \leqq \mathrm{k} \operatorname{gr}(J, E)$.

(2) If $\left(x_{1}, \cdots, x_{n}\right)=J$ then $\mathrm{k} \operatorname{gr}(J, E) \leqq n$.

(3) $\mathrm{c} \operatorname{gr}(J, E) \leqq \mathrm{k} \operatorname{gr}(J, E)$.

An immediate consequence of Proposition 1 is that the length of an $R$ sequence in an ideal $I$ is bounded by the minimum number of generators

Received by the editors September 23, 1971.

AMS (MOS) subject classifications (1970). Primary 13C99; Secondary 13A99, $13 \mathrm{D} 99$.

Key words and phrases. $R$-sequences, grade, Koszul complex, faithfully flat modules, coherent rings, Ext.

${ }^{1}$ The author wishes to thank his thesis advisor, Professor John Eagon, for his help in preparing this paper.

(C) American Mathematical Society 1973 
of $I$. It is easy to see that for $I$ finitely generated $\mathrm{kgr}(I, E)=0$ is equivalent to the statement: there exists $l_{0} \neq 0, l_{0}$ in $E$, so that $I l_{0}=0$.

For $u$ in $I, u$ not a zero divisor on $E$, one can easily verify that $\mathrm{c} \operatorname{gr}(I, E / u E) \leqq \mathrm{c} \operatorname{gr}(I, E)-1$. If the quantities involved are finite, one has equality if and only if $u$ is the first term of a longest maximal $R$-sequence on $E$ in $I$. By applying Proposition 2 and Theorem 4 of Chapter 8 of [7] one gets $\mathrm{k} \operatorname{gr}(I, E)=\mathrm{k} \operatorname{gr}(I, E / u E)+1$. The behaviors under polynomial extensions are covered in the next two propositions.

Proposition 2. Let $S$ be a faithfully flat extension of $R, I$ an ideal on $R, E$ an $R$-module with $I E \neq E$, then

(1) $\left(I \otimes_{R} S\right)\left(E \otimes_{R} S\right) \neq\left(E \otimes_{R} S\right)$, and

(2) $\mathrm{k} \operatorname{gr}(I, E)=\mathrm{k} \operatorname{gr}\left(I \otimes_{R} S, E \otimes_{R} S\right)$.

Proof. The key observation is that for $v_{1}, \cdots, v_{n}$ in $I \otimes_{R} S$, there exist $t_{1}, \cdots, t_{m}$ in $I$ so that $\left(v_{1}, \cdots, v_{n}\right) \subseteq\left(t_{1} \otimes 1, \cdots, t_{m} \otimes 1\right)$. The remainder of the proof follows from properties of the Koszul complex and faithfully flat modules [2].

If $\mathrm{c} \operatorname{gr}(I, E)=0$ and $\mathrm{kgr}(I, E) \neq 0$ then for $S=R[x]$ and $a_{0}, \cdots, a_{n}$ in $I$ so that $g\left(a_{0}, \cdots, a_{n} \mid E\right) \neq 0$, one has $a_{0}+a_{1} x+\cdots+a_{n} x^{n}$ is not a zero divisor on $E \otimes_{R} S$. Thus $\mathrm{c} \operatorname{gr}\left(I \otimes_{R} S, E \otimes_{R} S\right)>0$. As an extension of this one has:

Proposition 3. If $\mathrm{c} \operatorname{gr}(I, E)=V<n=\mathrm{k} \operatorname{gr}(I, E)$, then for some positive integer $t$ and for $S=R\left[x_{1}, \cdots, x_{t}\right]$ one has

$$
\mathrm{c} \operatorname{gr}\left[I \otimes_{R} S, E \otimes_{R} S\right]=\mathrm{kgr}\left[I \otimes_{R} S, E \otimes_{R} S\right]=n .
$$

For $I \subseteq J \subseteq \operatorname{Rad} I$, it is easy to see that $\mathrm{k} \operatorname{gr}(I, E)=\mathrm{k} \operatorname{gr}(J, E)$.

Let $k$ be a field, let $u_{f}$ be an indeterminate for $f \neq 0, f$ in the ideal generated by $x_{0}, \cdots, x_{n}$ of $k\left[x_{0}, \cdots, x_{n}\right]$. Let $T=k\left[x_{0}, \cdots, x_{n}\right]\left[\left\{u_{f}\right\}\right]$. Let $I_{1}$ and $I_{2}$ be ideals generated by objects of the form $f u_{f}$ and $u_{f} f_{g}$ respectively. Let $R=T /\left(I_{1}+I_{2}\right)$ and $I=\left(x_{0}, x_{1}\right)$, where $x_{0}$ represents $x_{0}+\left(I_{1}+I_{2}\right) . \mathrm{c} \operatorname{gr}(I, R)=0$ and $\mathrm{k} \operatorname{gr}(I, R) \neq 0$. Let $K\left(a_{1}, \cdots, a_{n}\right)$ denote the Koszul complex determined by $a_{1}, \cdots, a_{n}$, and let $H_{i}\left(K\left(a_{1}, \cdots, a_{n}\right)\right)$ denote the $i$ th homology module of $K\left(a_{1}, \cdots, a_{n}\right)$. One has

$$
H_{0}\left(K\left(a_{1}, \cdots, a_{n}\right)\right)=R /\left(a_{1}, \cdots, a_{n}\right),
$$

and the exact sequence:

$$
0 \longrightarrow H_{1}\left(K\left(x_{0}, x_{1}\right)\right) \longrightarrow R /\left(x_{0}\right) \stackrel{x_{1}}{\longrightarrow} R /\left(x_{0}\right) \longrightarrow R /\left(x_{0}, x_{1}\right) \longrightarrow 0 .
$$

Since $x_{1}$ is a zero divisor on $R /\left(x_{0}\right), H_{1} K\left(x_{0}, x_{1}\right) \neq 0$. So $\mathrm{kgr}(I, R)=1$. In like manner one can show $\mathrm{k} \operatorname{gr}\left(\left(x_{0}, x_{1}, \cdots, x_{n}\right), R\right)=1$. 
For another example, let $k$ be a field and $T=k\left[y, x_{1}, \cdots, x_{n}\right]$. For $f \neq 0, f$ a polynomial in $x_{1}, x_{2}, \cdots, x_{n}$ without constant term, adjoin an indeterminate $u_{f}$ to $T$ forming $T_{1}=T\left[\left\{u_{f}\right\}\right]$. Let $I_{1}$ and $I_{2}$ be ideals generated by elements of the forms $u_{f} u_{g}$ and $u_{f}(y-f)$ respectively. Let $R=T_{1} /\left(I_{1}+I_{2}\right)$ and let $I=\left(y, x_{1}, \cdots, x_{n}\right) . n \leqq \mathrm{cgr}(I, R) \leqq \mathrm{k} \operatorname{gr}(I, R) \leqq n+1$ so $n-1 \leqq$ $\mathrm{k} \operatorname{gr}(I, R /(y)) \leqq n$ but $\mathrm{c} \operatorname{gr}(I, R /(\boldsymbol{y}))=0$.

2. Homological representation. Rees [8] and [9] proved that in a Noetherian ring $R, \mathrm{c} \operatorname{gr}(I, E)=n$ provided $n$ is the least integer so that $\operatorname{Ext}^{n}(R / I, E) \neq 0$. Denote by $\mathrm{r} \operatorname{gr}(I, E)$ the least integer $n$ such that $\mathrm{Fxt}_{R}^{n}(R / I, E) \neq 0$. Rees [9] proves $\mathrm{c} \operatorname{gr}(I, E) \leqq \mathrm{r} \operatorname{gr}(I, E)$ and for $u$ in $I, u$ not a zero divisor on $E, \mathrm{r} \operatorname{gr}(I, E)=1+\mathrm{r} \operatorname{gr}(I, E / u E)$.

It is easy to see that $\operatorname{rgr}(I, E)=0$ is equivalent to the statement: There exist $l_{0} \neq 0, l_{0}$ in $E$, so that $I l_{0}=0$. For $I$ finitely generated both are equivalent to $\mathrm{k} \operatorname{gr}(I, E)=0$. For $I$ finitely generated and $\mathrm{c} \operatorname{gr}(I, E)=\mathrm{k} \operatorname{gr}(I, E)$ one can easily see that $\mathrm{r} \operatorname{gr}(I, E)=\mathrm{k} \operatorname{gr}(I, E)$. This is not true for infinitely generated ideals. For $k$ a field and

$$
R=k\left[x_{1}, x_{2}, \cdots, x_{n}, \cdots\right] /\left(x_{1}^{1}, x_{2}^{2}, x_{3}^{3}, \cdots, x_{n}^{n}, \cdots\right)
$$

and $I=\left(x_{2}, x_{3}, \cdots, x_{n}, \cdots\right), \mathrm{c} \operatorname{gr}(I, R)=\mathrm{k} \operatorname{gr}(I, R)=0$ but $\mathrm{r} \operatorname{gr}(I, R)>0$.

PROPOSITION 4. For I finitely generated, one has $\mathrm{k} \operatorname{gr}(I, E)=n$ where $n$ is the least integer such that $\operatorname{Ext}_{R}^{n}(R / I, E \infty) \neq 0$. Here $E \infty$ is the direct sum of countably many copies of $E$. Further $\mathrm{k} \operatorname{gr}(I, E) \leqq \mathrm{r} \operatorname{gr}(I, E)$.

Proof. The last remark follows from the first and $E \infty=E \oplus E \infty$. By Proposition 3, there is an integer $s$ so that for $S=R\left[x_{1}, \cdots, x_{s}\right]$ one has

$$
\begin{aligned}
\mathrm{kgr}(I, E) & =\mathrm{kgr}\left[\left(I \otimes_{R} S\right),\left(E \otimes_{R} S\right)\right] \\
& =\mathrm{c} \operatorname{gr}\left(I \otimes_{R} S, E \otimes_{R} S\right)=\mathrm{r} \operatorname{gr}\left(I \otimes_{R} S, E \otimes_{R} S\right) .
\end{aligned}
$$

By the homological identity

$$
\operatorname{Ext}_{R}^{n}\left(R / I, E \otimes_{R} S\right) \cong \operatorname{Ext}_{S}^{n}\left[S / I \otimes_{R} S, E \otimes_{R} S\right],
$$

the result follows. For a proof of the identity see Theorem 3.1 of [3].

A ring $R$ is called coherent if every finitely generated ideal is finitely presented. Properties of coherent rings are discussed in [4]. The next proposition was suggested to me by $M$. Hockster who gave a proof of the first part. As a preliminary, note that for $R$ a ring and $k$ an integer $0 \rightarrow K_{1} \rightarrow R^{k} \rightarrow K \rightarrow 0$ exact, the map $\operatorname{Hom}\left(R^{k}, E \infty\right) \rightarrow \operatorname{Hom}(K, E \infty)$ assigns to $\alpha$ in $\operatorname{Hom}\left(R^{k}, E \infty\right)$ a certain matrix map in $\operatorname{Hom}\left(K_{1}, E\right)$.

Proposition 5. For I finitely generated:

(1) If $\mathrm{k} \operatorname{gr}(I, E)=1$, then $\mathrm{r} \operatorname{gr}(I, E)=1$.

(2) If $R$ is coherent and $\mathrm{k} \operatorname{gr}(I, E)=n$ then $\mathrm{r} \operatorname{gr}(I, E)=n$. 
Proof. For $I$ finitely generated and $R$ coherent one has a long exact sequence

$$
0 \rightarrow K \rightarrow F_{n-1} \rightarrow F_{n-2} \rightarrow \cdots \rightarrow F_{1} \rightarrow I \rightarrow 0
$$

with $K$ finitely generated and $F_{i}$ free and finitely generated. This gives

$$
0 \rightarrow K \rightarrow F_{n-1} \rightarrow F_{n-2} \rightarrow \cdots \rightarrow F_{1} \rightarrow R \rightarrow R / I \rightarrow 0 .
$$

One has $\operatorname{Ext}^{n}(R / I, E \infty) \neq 0$ where $n=\mathrm{k} \operatorname{gr}(I, E)$. If $\mathrm{r} \operatorname{gr}(I, E) \neq n$ then one has $\operatorname{Ext}^{n}(R / I, E)=0$. Thus $\operatorname{Ext}^{1}(K, E)=0$ and $\operatorname{Ext}^{1}(K, E \infty) \neq 0$. For $F_{n-1}=$ $R^{k}$, and $0 \rightarrow K^{\prime} \rightarrow F^{k} \rightarrow K \rightarrow 0$ one gets, for $K^{\prime}$ finitely generated,

$$
\operatorname{Hom}\left(R^{k}, E\right) \stackrel{t}{\longrightarrow} \operatorname{Hom}\left(K^{\prime}, E\right) \longrightarrow \operatorname{Ext}(K, E)=0 .
$$

Thus $t$ is onto. Now, this says every homomorphism $K^{\prime} \rightarrow E$ is given by the $\operatorname{map}\left(v_{1}, \cdots, v_{k}\right) \rightarrow \sum v_{i} l_{i}$ for $\left(l_{1}, \cdots, l_{k}\right)$ is a fixed element of $E^{k}$. Likewise

$$
\operatorname{Hom}\left(F^{k}, E \infty\right) \stackrel{\gamma}{\longrightarrow} \operatorname{Hom}\left(k^{\prime}, E \infty\right) \longrightarrow \operatorname{Ext}(K, E \infty) \longrightarrow 0
$$

so $\gamma$ is not onto, thus there is a homomorphism $g: K^{\prime} \rightarrow E \infty$ which is not a matrix map. Since $K^{\prime}$ is finitely generated, $\operatorname{Im} g \subseteq E^{m}$ some $m$, so $g$ is determined by its projections $g_{j}, g_{j}: K^{\prime} \rightarrow E$ by $g_{j}=g \circ \pi_{j}, \pi_{j}: E \infty \rightarrow E$ ( $j$ th projection) $j=1, \cdots, m$. Thus $g_{j}$ is determined by $\left(l_{1}^{(j)}, l_{2}^{(j)}, \cdots, l_{k}^{(j)}\right)$. Thus $g$ is given by a matrix. It is noted that for part (1) one does not need that $R$ is coherent.

\section{BIBLIOGRAPHY}

1. S. F. Barger, Generic perfection and the theory of grade, Doctoral Thesis, University of Minnesota, Minneapolis, Minn.

2. N. Bourbaki, Éléments de mathématique. Fasc. XXVII. Algèbre commutative, Actualitiés Sci. Indust., no. 1290, Hermann, Paris, 1961. MR 36 \#146.

3. H. Cartan and S. Eilenberg, Homological algebra, Princeton Univ. Press, Princeton, N.J., 1956. MR 17, 1040.

4. S. U. Chase, Direct products of modules, Trans. Amer. Math. Soc. 97 (1960), 457-473. MR 22 \#11017.

5. I. Kaplansky, Commutative rings, Allyn and Bacon, Boston, Mass., 1970. MR 40 \#7234.

6. D. G. Northcott, Grade sensitivity and generic perfection, Proc. London Math. Soc. (3) 20 (1970), 597-618. MR 42 \#7652.

7. - Lessons on rings, modules and multiplicities, Cambridge Univ. Press, London, 1968. MR 38 \#144.

8. D. Rees, The grade of an ideal or module, Proc. Cambridge Philos. Soc. 53 (1957), 28-42. MR 18, 637.

9. - A theorem of homological algebra, Proc. Cambridge Philos. Soc. 52 (1956), 605-610. MR 18, 277.

Department of Mathematics, Youngstown State University, Youngstown, OHIO 44503 